\title{
Avoiding harm: Central venous access in Kartagener syndrome and other vascular anomalies
}

\author{
Brad Snodgrass MD, FACP, Victoria Chu MS, MBA
}

\begin{abstract}
Placement of internal jugular catheters is more likely to be complicated if a left-sided approach is used, assuming normal anatomy. Kartagener syndrome is the sine qua non of sidedness confusion and results in cognitive challenges that increase the risk of adverse patient outcomes. The altered anatomy can cause profound disorientation from our usual processes. In normal circumstances the right-sided approach is used for placement of internal jugular catheters, but in Kartagener syndrome the left-sided approach should be preferred. Surgical volume and use of ultrasound guided techniques are positively correlated with better outcomes. Clinical experience may be a detriment to performance. Knowledge of these issues will help clinicians maintain vigilance and avoid error.
\end{abstract}

Keywords: Kartagener syndrome, central venous access, superior vena cava, landmark technique, internal jugular vein catheterization cognitive bias

\section{CASE}

A patient with known Kartagener syndrome presented with septic shock secondary to a perforated viscus. In preparing for emergency laparotomy, a central venous catheter was placed at the bedside using the right internal jugular approach. The catheter position was checked with anterior-posterior portable chest radiography, which was interpreted as its being in the superior vena cava (Figure 1). Unfortunately, subsequent events showed that there had been a right carotid artery injury with an arteriovenous fistula demonstrated between the internal carotid artery and the internal jugular vein. The patient also received norepinephrine through the catheter. A right hemispheric carotid distribution stroke was diagnosed during the postoperative course. The fistula was

Corresponding author: Brad Snodgrass

Contact Information: Bradsnodgrass@gmail.com DOI: 10.12746/swrccc.v9i41.939

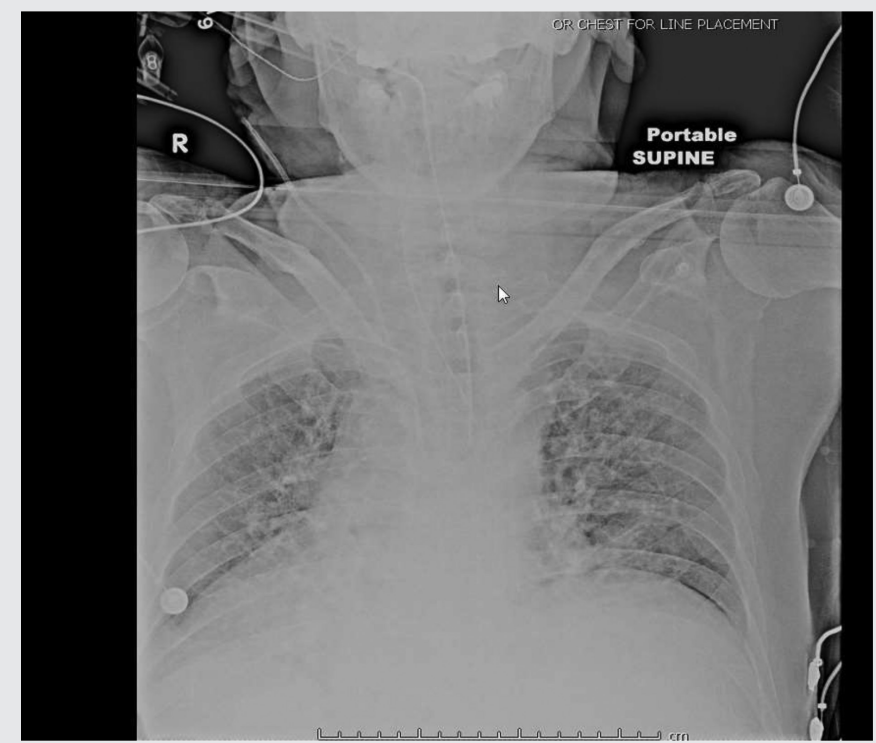

Figure 1. Post procedure film showing the central venous catheter overlying the right mediastinum.

repaired with a stent, and after a prolonged hospitalization, he was discharged with residual severe left hemiplegia. 


\section{Discussion}

Most patients have anatomic findings that do not challenge our usual and customary thinking processes. In these cases, we proceed with a cognitive process that is comfortable and automatic, akin to System 1 thinking described by Daniel Kahneman, e.g., driving a car on an empty road. ${ }^{1}$ Anatomic anomalies can confound our most basic assumptions, such as sidedness, single or paired structures, and physiologic principles. Our ingrained habits may render us unable to appreciate the need for System 2 thinking, which is complex, requiring deliberative cognitive work, e.g., parking a car in a narrow space. ${ }^{1}$ What seems simple and unchallenging may be surprisingly complex and disorienting. For example, someone who is accustomed to driving in the United States (US) will be required to do extra cognitive work while driving in the United Kingdom, i.e. the performance of the US driver will likely be constrained by ingrained habits. Likewise, when presented with a chest radiograph showing a right-sided heart silhouette, based on our usual expectations of normal anatomy, we are likely to believe that the film has been accidentally reversed, and very often we would be correct in this assumption. Even the patient may be unaware of the anomaly. The patient above did not know he had Kartagener syndrome until a chest $\mathrm{x}$-ray was performed when he was 36-years-old. The technician was accused of mishandling the film during production, but after a careful review she was proven correct (verbal communication from the patient).

In the case above, the catheter position was interpreted by an experienced radiologist as being in the superior vena cava (SVC), but this most likely could only be true if it had been located in the left hemithorax. The position of the SVC in Kartagener syndrome should be on the left, opposite the cardiac silhouette. But even this assertion may not be correct in all cases. Some patients, with otherwise normal anatomy, have an SVC on the left, either as a paired, dual-sided structure or as a single structure. Persistent left superior vena cava is present in $0.5-2 \%$ of the general population. An isolated left superior vena cava is even more rare, present in $0.09-0.13 \% .^{2}$ Presumably a patient with Kartagener syndrome might have a dual anomaly, with an SVC on the right, either as a paired, bilateral drainage system or as a single structure, but this combination would be truly rare.

Physicians spend many years acquiring the knowledge, skills, and judgement necessary to provide the best possible care to patients. We are routinely measured on the outcomes of our interventions. On procedural services, clinical performance has been correlated with surgical volume. ${ }^{3}$ But quality outcomes may be inversely related to years of experience, perhaps through decreasing cognitive capacity and reduced ability to adjust to novelty. ${ }^{4}$ However, physician age may be somewhat less important as an individual factor than the overall clinical setting, e.g., isolated versus tertiary centers and low versus high volume centers. ${ }^{5}$ Our daily practice is informed by regular exposure to normal examples in anatomy and physiology, or by variations that are of no major import. For example, it is accepted practice that when attempting an internal jugular catheter, we use the right-sided approach, and we have expectations of typical radiological findings to assure accuracy of placement. With novel situations, such as Kartagener syndrome, and other causes of unusual anatomy, our practice should be deliberative, both in performance and expectation of confirmatory data.

The internal jugular vein approach is complicated by internal carotid artery injury in up to $5.9 \%$ of cases when using the landmark technique. ${ }^{6}$ Malposition of internal jugular catheters have been demonstrated in up to $14 \%$ of patients, even in experienced hands. ${ }^{7}$ Schummer et al. prospectively studied 1,794 central line catheterizations by experienced providers using the landmark technique and found that $6.7 \%$ of the catheter tips were intravenously malpositioned, with left internal jugular catheters conferring the highest risk. ${ }^{8}$ Overall, there were $12.3 \%$ adverse events. The internal jugular approach resulted in arterial punctures $4.4 \%$ of the time.$^{8}$ In the case above, the right-sided approach was equivalent to a left-sided approach (in someone with normal anatomy), thus seemingly increasing the likelihood of the outcome. A left-sided approach would have been more appropriate. Additionally, the expected catheter position on $\mathrm{x}$-ray should have been on the left side. 
A post procedure chest radiograph has been questioned as a potential waste of resources with right internal jugular catheter placement. ${ }^{9,10}$ Lessnau found no significant complications in a series of 100 consecutive cases of right-sided internal jugular catheters, without mention of whether imaging guiding studies were used. ${ }^{9}$ Lucey et al. ${ }^{10}$ studied 621 imaging guided catheter placements in 489 patients and could not find a benefit for routine post procedure radiography. However, Gladwin et al. ${ }^{7}$ concluded that post procedure $x$-ray is needed based on the high rate of unexpected catheter malposition in their study; it is unclear if ultrasound or landmark techniques were used. Patients with known vascular anomalies might have been excluded from the above trials. But some anomalies are unrecognized or, if known, are underappreciated. The chest $\mathrm{x}$-ray could have been an earlier clue to the misadventure in the case above.

Another important point of interest in the Schummer study is that it included only procedures performed by the landmark technique, as "experienced personnel frequently reject ultrasound guidance, on the basis that their expertise renders this technique superfluous." Use of real-time guidance ultrasound has been shown to significantly reduce the likelihood of catheter misplacement. ${ }^{11}$

Most clinicians have had few patients with altered SVC anatomy, particularly Kartagener syndrome. Years of experience may be inversely proportional to optimal performance. System 1 thinking is familiar and automatic. Anatomic anomalies present a challenge to clinical performance and require diligent System 2 cognition, which increases the likelihood of error. Knowledge of the anatomic variations will promote vigilance and improve bedside reasoning. Awareness of pitfalls in the surgical approach, particularly when catheters are placed by landmark techniques and in emergency settings, may help avoid adverse outcomes. In addition, the expectations of post procedure data require diligent communication among all caregivers. The crucial combination of knowledge, skill, reasoning, judgement, and teamwork required in all clinical encounters is even more paramount when confronted with unusual disorders. The difficulties illustrate what it means to attend to the patient.

\section{HIGHLIGHTS}

1. When confronted with atypical anatomy, we are disoriented from our customary processes.

2. Unusual anatomy requires cognitive work that is deliberative.

3. When seeking confirmatory data, it is imperative to maintain heightened vigilance.

4. Communication within the entire care team is paramount to avoid error.

5. The landmark technique is more likely to result in catheter misplacement than ultrasound guidance.

6. Internal jugular catheters are more likely to be complicated than subclavian catheters.

7. Left internal jugular catheters are most likely to result in carotid artery injury.

\section{ACKNOWLEDGEMENTS}

We thank Joel Velsco, PhD and P. Carter Snodgrass, MD, MBA for fruitful discussions and review of the manuscript. We thank Amit Bushan, MBA for his expert technical assistance.

Article citation: Snodgrass B, Chu V. Avoiding harm: Central venous access in Kartagener syndrome and other vascular anomalies. The Southwest Respiratory and Critical Care Chronicles 2021;9(41):40-43

From: Department of Internal Medicine, Texas Tech University Health Sciences Center, Lubbock, Texas

Submitted: 9/21/2021

Accepted: 9/29/2021

Reviewer: Kenneth Nugent MD

Conflicts of interest: none

This work is licensed under a Creative Commons Attribution-ShareAlike 4.0 International License.

\section{REFERENCES}

1. Kahneman D. The Characters of the Story: Two systems. Thinking Fast and Slow. Farrar, Straus and Giroux. 2011. p. 20-26. 
2. Sheikh AS, Mazhar S. Persistent left superior vena cava with absent right superior vena cava; review of the literature and clinical implications. Echocardiography 2014;31(5): 674-679.

3. Halm EA, Lee C, Chassin MR. Is volume related to outcome in health care? a systematic review and methodologic critique of the literature. Ann Intern Med 2002;137(6):511-520.

4. Choudry NK, Fletcher RH, Soumerai SB. Systematic review: the relationship between clinical experience and quality of health Care. Ann Intern Med 2005;142:260-273.

5. Wenghofer EF, Williams AP, Klass DJ. Factors affecting physician performance: implications for performance improvement and governance. Health Policy 2009 Nov: 5(2): e141-e160.

6. Reuber M, Dunkley LA, Turton EPL, et al. Stroke after internal jugular venous cannulation. Acta Neurol Scand 2002: 105:235-239.

7. Gladwin MT, Slonim A, Landucci DL, et al. Cannulation of the internal jugular vein: is postprocedural chest radiography always necessary? Crit Care Med. 1999 Sep; 27(9):1819-23.

8. Schummer W, Schummer C, Rose N, et al. Mechanical complications and malpositions of central venous cannulations by experienced operators: A prospective study of 1794 catheterizations in critically ill patients. Intensive Care Med 2007;(33):1055-1059.

9. Lessnau K. Is chest radiography necessary after uncomplicated insertion of a triple-lumen catheter in the right internal jugular vein using the anterior approach? Chest 2005;127(1): 220-223.

10. Lucey B, Varghese JC, Haslam P, et al. Routine chest radiographs after central line insertion: mandatory postprocedural evaluation or unnecessary waste of resources? Cardiovasc Intervent Radiol 1999;22(5):381-384.

11. Hind D, Calvert N, McWilliams R, et al. Ultrasonic locating devices for central venous cannulation: meta-analysis. BMJ 2003;327(7411):361. https://doi.org/10.1136/bmj.327. 7411.3615 